\title{
PERIODS OF GENERIC TORSORS OF GROUPS OF MULTIPLICATIVE TYPE
}

\author{
WANSHUN WONG
}

\begin{abstract}
In this paper we establish different ways to compute the period of a generic torsor of a group of multiplicative type. We also study the relationships among the periods of generic torsors of $Q, T$ and $W$, where $Q$ is a group of multiplicative type, $T$ is the maximal subtorus of $Q$ and $W$ is the maximal finite quotient group of $Q$.
\end{abstract}

\section{INTRODUCTION}

Let $F$ be our fixed base field, and let $Q$ be a smooth commutative linear algebraic group over $F$. For every field extension $K / F$, there is a bijective correspondence between the set of isomorphism classes of $Q$-torsors (principal homogeneous spaces) over $K$ and the first Galois cohomology group $H^{1}(K, Q)$. The period of a $Q$-torsor is the order of the corresponding element in $H^{1}(K, Q)$. Note that the period of a $Q$-torsor is always finite as $H^{1}(K, Q)$ is a torsion group.

A short exact sequence of linear algebraic groups

$$
1 \longrightarrow Q \longrightarrow P \stackrel{\phi}{\longrightarrow} S \longrightarrow 1
$$

where $P$ is connected, reductive and special, is called a resolution of $Q$. Since we can embed $Q$ into $\mathbf{G L}_{n}$ for some positive integer $n$, resolutions of $Q$ exist. The generic fiber of the morphism $\phi: P \rightarrow S$ is a $Q$-torsor over the function field $F(S)$ of $S$, moreover it is a generic $Q$-torsor (see [1, section 6] and [5]). In this paper by a generic $Q$-torsor, we mean a generic $Q$-torsor that arises from a resolution of $Q$ as above.

When $Q$ is an algebraic torus, the period of a generic $Q$-torsor is studied in [7]. Based on the technique developed in [7], we generalize the results to the case where $Q$ is a smooth group of multiplicative type (see Corollary 4.3). We also study in Section 5 the relationships among the periods of generic torsors of $Q, T$ and $W$, where $T$ is the maximal subtorus of $Q$ and $W$ is the maximal finite quotient group of $Q$. In Section 6 we give an application of our results.

\section{Periods of Generic Torsors}

Proposition 2.1. Let $Q$ be a smooth commutative linear algebraic group over $F$. Assume that one of the following conditions hold:

(a) $F$ is infinite.

(b) The connected component $Q^{0}$ of $Q$ is reductive.

2000 Mathematics Subject Classification. 11E72, $20 \mathrm{G} 15$.

Key words and phrases. Generic Torsor, Galois Cohomology, Groups of Multiplicative Type. 
Then the period of a generic $Q$-torsor is divisible by the period of any $Q$-torsor over any field extension $K / F$.

Proof. The proof is essentially the same as that of [7, Proposition 1.1]. Suppose that $F$ is a finite field. Then by assumption the connected component $Q^{0}$ of $Q$ is reductive.

Claim: The natural map $H^{1}(F, Q) \rightarrow H^{1}(F((t)), Q)$ is injective.

The following argument is suggested by Philippe Gille. Consider the exact sequence of linear algebraic groups over $F$

$$
1 \longrightarrow Q^{0} \longrightarrow Q \longrightarrow \pi_{0}(Q) \longrightarrow 1
$$

where $\pi_{0}(Q)$ is finite étale. It induces the following commutative diagram

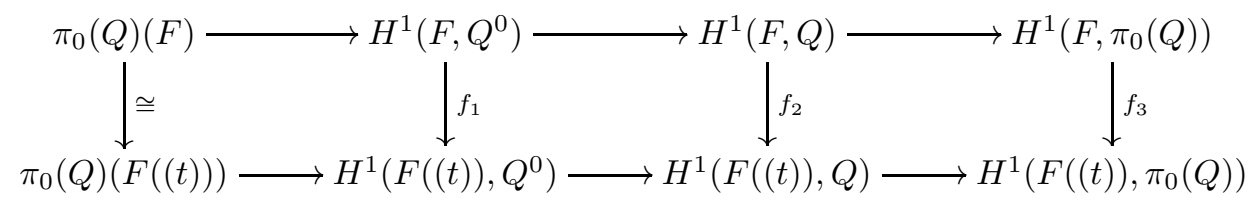

By [6, Theorem I.1.2.2] $f_{1}$ is injective. Notice that $f_{3}$ is injective also, hence by four lemma $f_{2}$ is injective.

It follows from the claim that we may replace $F$ by $F((t))$ and assume that our base field is infinite.

Let $E \rightarrow \operatorname{Spec} F(S)$ be a generic $Q$-torsor that arises from a resolution of $Q$

$$
1 \longrightarrow Q \longrightarrow P \stackrel{\phi}{\longrightarrow} S \longrightarrow 1 \text {. }
$$

We denote by $[E]$ the class of $Q$-torsor $E \rightarrow \operatorname{Spec} F(S)$ in $H^{1}(F(S), Q)$, and $[P]$ the class of $\phi: P \rightarrow S$ in the first étale cohomology group $H_{e ́ t}^{1}(S, Q)$. Then $[E]$ is equal to the image of $[P]$ under the natural map $H_{e ́ t}^{1}(S, Q) \rightarrow H_{e ́ t}^{1}(\operatorname{Spec} F(S), Q)=$ $H^{1}(F(S), Q)$. As $H^{1}(F(S), Q)$ is the colimit of $H_{e ́ t}^{1}(U, Q)$ over all nonempty open subsets $U \subseteq S$, there exists a nonempty open $U$ such that the order of $[P]_{U}$, where $[P]_{U}$ is the image of $[P]$ in $H_{e ́ t}^{1}(U, Q)$, is equal to the order of $[E]$.

Let $R$ be a $Q$-torsor over $K$, where $K$ is a field extension over $F$. The above resolution induces a long exact sequence

$$
P(K) \longrightarrow S(K) \longrightarrow H^{1}(K, Q) \longrightarrow H^{1}(K, P) \text {. }
$$

Since $P$ is special, $H^{1}(K, P)$ is trivial. Thus there exists a point $s \in S(K)$ such that the class $[R]$ of $R$ in $H^{1}(K, Q)$ is equal to the class of the $Q$-torsor $\phi^{-1}(s)$. Notice that $K$ is infinite, it follows from [2, Corollary 18.3] that the set $K$-rational points $P(K)$ is dense in $P$. This implies that $\phi(P(K))$ is dense in $S$ because $\phi: P \rightarrow S$ is surjective. Hence $\phi(P(K)) \cdot s$ is also dense in $S$ and there exists a point $s^{\prime} \in U \cap(\phi(P(K)) \cdot s)$. From the long exact sequence $(1)$ the class of $\phi^{-1}\left(s^{\prime}\right)$ is equal to that of $\phi^{-1}(s)$. Therefore by replacing $s$ by $s^{\prime}$ we may assume $s \in U(K)$. Then the morphism $s: \operatorname{Spec} K \rightarrow U \rightarrow S$ induces the following map

$$
\begin{array}{ccccc}
H_{e ́ t}^{1}(S, Q) & \longrightarrow & H_{e ́ t}^{1}(U, Q) & \longrightarrow & H_{e ́ t}^{1}(\operatorname{Spec} K, Q)=H^{1}(K, Q) \\
{[P]} & \longmapsto & {[P]_{U}} & \longmapsto & {\left[\phi^{-1}(s)\right]=[R]}
\end{array}
$$

It follows that the order of $[R]$ divides the order of $[P]_{U}$, which is equal to the order of $[E]$. 
By Proposition 2.1 all generic $Q$-torsors have the same period. In other words, the period of a generic $Q$-torsor depends only on $Q$, and we denote this number by $e(Q)$. Proposition 2.1 also shows that $e(Q)$ is the smallest positive integer that annihilates $H^{1}(K, Q)$ for every field extension $K / F$. Thus $e(Q)$ can be viewed as the "exponent" of the first Galois cohomology functor

$$
H^{1}(-, Q): \text { Fields } / F \longrightarrow A b
$$

where Fields $/ F$ is the category of field extensions of $F$ and field homomorphisms over $F$, and $A b$ is the category of abelian groups.

\section{Preliminaries on Groups of Multiplicative Type}

Unless otherwise specified, all groups of multiplicative type in this paper are over $F$ and are smooth, and they all split over a finite Galois extension $L / F$. Let $G$ be the Galois group $\operatorname{Gal}(L / F)$, and let $Q^{*}$ be the character $G$-module of a group of multiplicative type $Q$ over $L$. Notice that the connected component of a smooth group of multiplicative type is a torus, which is reductive, hence we can apply Proposition 2.1.

Let

$$
\theta: \quad 1 \longrightarrow Q \longrightarrow R \stackrel{g}{\longrightarrow} S \longrightarrow 1
$$

be an exact sequence of groups of multiplicative type, where $S$ is a torus.

For any subgroup $H$ of $G$, we have the following pairing:

$$
H^{0}\left(H, \operatorname{Hom}_{\mathbb{Z}}\left(Q^{*}, \mathbb{Z}\right)\right) \otimes H^{1}\left(H, S^{*}\right) \rightarrow \operatorname{Ext}_{G}^{1}\left(Q^{*}, S^{*}\right) .
$$

First we identify

$$
H^{0}\left(H, \operatorname{Hom}_{\mathbb{Z}}\left(Q^{*}, \mathbb{Z}\right)\right)=\operatorname{Hom}_{G}\left(Q^{*}, \mathbb{Z}[G / H]\right)=\operatorname{Ext}_{G}^{0}\left(Q^{*}, \mathbb{Z}[G / H]\right)
$$

and

$$
H^{1}\left(H, S^{*}\right)=\operatorname{Ext}_{H}^{1}\left(\mathbb{Z}, S^{*}\right)=\operatorname{Ext}_{G}^{1}\left(\mathbb{Z}[G / H], S^{*}\right) .
$$

Then we use the composition product for Ext,

$$
\operatorname{Ext}_{G}^{0}\left(Q^{*}, \mathbb{Z}[G / H]\right) \otimes \operatorname{Ext}_{G}^{1}\left(\mathbb{Z}[G / H], S^{*}\right) \rightarrow \operatorname{Ext}_{G}^{1}\left(Q^{*}, S^{*}\right) .
$$

The following proposition is a slight generalization of [7, Proposition 2.1].

Proposition 3.1. The following are equivalent:

(a) For every field extension $K / F$, the homomorphism $g_{K}: R(K) \rightarrow S(K)$ is surjective.

(b) The generic point $\xi$ of $S$ in $S(F(S))$ is in the image of $g_{F(S)}$.

(c) The exact sequence $\theta$ has a rational splitting.

(d) There exists a commutative diagram of morphisms of groups of multiplicative type with splitting field $L / F$ :

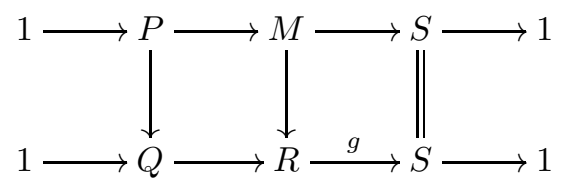

with exact rows and $P$ is a quasi-split torus. 
(e) The class of the exact sequence $\theta$ in $\operatorname{Ext}_{G}^{1}\left(Q^{*}, S^{*}\right)$ lies in the image of the map

$$
\varphi: \coprod_{H<G} H^{0}\left(H, \operatorname{Hom}_{\mathbb{Z}}\left(Q^{*}, \mathbb{Z}\right)\right) \otimes H^{1}\left(H, S^{*}\right) \rightarrow \operatorname{Ext}_{G}^{1}\left(Q^{*}, S^{*}\right)
$$

induced by the pairing (2), where the coproduct is taken over all subgroups $H$ of $G$.

If $S$ is coflasque, these conditions are also equivalent to

(f) The exact sequence $\theta$ splits.

Proof. (a) $\Rightarrow(\mathrm{b})$ : Obvious.

(b) $\Rightarrow(\mathrm{c})$ : Let $x \in R(F(S))$ be a point such that $g_{F(S)}(x)=\xi$. The commutative diagram

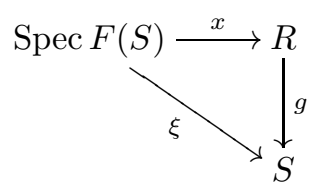

implies that there is a nonempty open subset $U \subseteq S$ together with the following commutative diagram

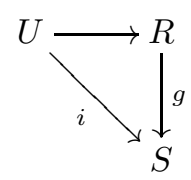

where $i: U \rightarrow S$ is the inclusion.

$(\mathrm{c}) \Rightarrow(\mathrm{d})$ : By assumption there exists a rational splitting $h: S--\rightarrow R$. Let $U$ be the domain of definition of $h$, and let $\Lambda$ be the lattice $L[U]^{\times} / L^{\times}$. The character lattice $S^{*}$ of $S$ is isomorphic to $L[S]^{\times} / L^{\times}$which is a sublattice of $\Lambda$, and the factor lattice $\Lambda / S^{*}$ is permutation by the proof of [3, Proposition 5]. Let $M$ and $P$ be the tori with character lattices $\Lambda$ and $\Lambda / S^{*}$ respectively.

The character group $R^{*}$ of $R$ is isomorphic to a subgroup of $L[R]^{\times}$(the subgroup of group-like elements), therefore there is a natural homomorphism $R^{*} \rightarrow$ $L[R]^{\times} / L^{\times}$. The morphism $h: U \rightarrow R$ induces a homomorphism $L[R]^{\times} / L^{\times} \rightarrow \Lambda$. The composition $R^{*} \rightarrow L[R]^{\times} / L^{\times} \rightarrow \Lambda$ then induces a morphism of groups of multiplicative type $M \rightarrow R$. Since $h$ is a splitting, by construction the composition $M \rightarrow R \rightarrow S$ is equal to the morphism $M \rightarrow S$ induced by the inclusion $S^{*} \longrightarrow \Lambda$.

$(\mathrm{d}) \Rightarrow(\mathrm{a})$ : For every $K / F, H^{1}(K, P)=0$ implies $M(K) \rightarrow S(K)$ is surjective, so $g_{K}$ is also surjective.

$(\mathrm{d}) \Leftrightarrow(\mathrm{e})$ : The diagram in (d) exists if and only if there is a quasi-split torus $P$ and a homomorphism $\alpha: Q^{*} \rightarrow P^{*}$ such that the class of the exact sequence $\theta$ lies in the image of the induced map $\alpha^{*}: \operatorname{Ext}_{G}^{1}\left(P^{*}, S^{*}\right) \rightarrow \operatorname{Ext}_{G}^{1}\left(Q^{*}, S^{*}\right)$. Since $P$ is quasi-split, $P^{*}$ is a direct sum of lattices of the form $\mathbb{Z}[G / H]$, where $H$ is a subgroup of $G$.

Consider the case $P^{*}=\mathbb{Z}[G / H]$. The map $\alpha$ is given by an element $a \in$ $H^{0}\left(H, \operatorname{Hom}_{\mathbb{Z}}\left(Q^{*}, \mathbb{Z}\right)\right)$. Also, we have shown that $\operatorname{Ext}_{G}^{1}\left(\mathbb{Z}[G / H], S^{*}\right)=H^{1}\left(H, S^{*}\right)$. Then the map $\alpha^{*}$ coincides with multiplication by $a$ with respect to the pairing $(2)$.

(f) $\Rightarrow(\mathrm{c})$ : Obvious. 
(e) $\Rightarrow$ (f) if $S$ is coflasque: Notice that $H^{1}\left(H, S^{*}\right)=0$ for every subgroup $H$ of $G$ because $S$ is coflasque. Therefore $\operatorname{im}(\varphi)$ is trivial, and it implies that $\theta$ is a split short exact sequence.

\section{MAin Result}

Let

$$
\chi: \quad 1 \longrightarrow Q \stackrel{\iota}{\longrightarrow} P \longrightarrow S \longrightarrow 1
$$

be a coflasque resolution of $Q$, i.e. $P$ is a quasi-split torus and $S$ is a coflasque torus. By [4, Proposition 1.3] coflasque resolutions of $Q$ exist.

Theorem 4.1. Let $f: Q \rightarrow Q^{\prime}$ be a morphism of groups of multiplicative type. Then the following are equivalent:

(a) f factors through $\iota: Q \rightarrow P$.

(b) $f$ factors through some quasi-split torus.

(c) The induced map $H^{1}(K, Q) \rightarrow H^{1}\left(K, Q^{\prime}\right)$ is the zero homomorphism for every field extension $K / F$.

Proof. It is clear that (a) $\Rightarrow(\mathrm{b}) \Rightarrow$ (c), so it suffices to prove (c) $\Rightarrow$ (a).

Assume (c) holds. Let $R$ be a group of multiplicative type defined by the following commutative diagram

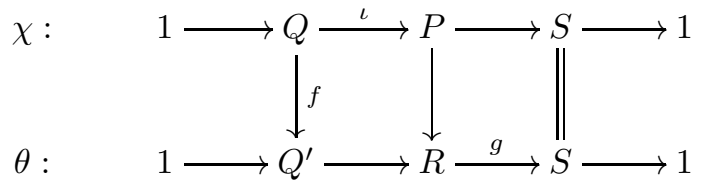

where the rows are exact. For every field extension $K / F$, the commutative diagram

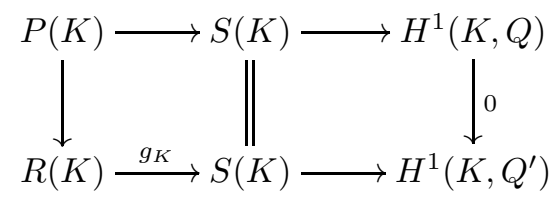

shows that $g_{K}: R(K) \rightarrow S(K)$ is surjective. Since $S$ is coflasque, by Proposition $3.1 \theta$ is a split short exact sequence. Then the result follows easily from the the following commutative diagram

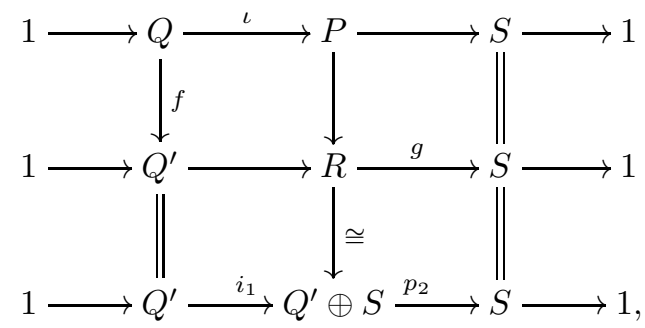

where $i_{1}$ and $p_{2}$ are canonical inclusion and projection respectively.

Remark 4.2. Note that $(\mathrm{b}) \Rightarrow$ (a) can be proved directly as follows (see [3, Lemma $4])$ : Suppose $f$ factors through a quasi-split torus $R$, our goal is to show that $f: Q \rightarrow R \rightarrow Q^{\prime}$ factors as $Q \stackrel{\iota}{\rightarrow} P \rightarrow R \rightarrow Q^{\prime}$. 
Consider the exact sequence

$$
\operatorname{Hom}_{G}\left(R^{*}, P^{*}\right) \stackrel{\iota^{*}}{\longrightarrow} \operatorname{Hom}_{G}\left(R^{*}, Q^{*}\right) \longrightarrow \operatorname{Ext}_{G}^{1}\left(R^{*}, S^{*}\right) .
$$

Since $R^{*}$ is a permutation module and $S^{*}$ is coflasque, $\operatorname{Ext}^{1}\left(R^{*}, S^{*}\right)$ is trivial. Hence $R^{*} \rightarrow Q^{*}$ factors as $R^{*} \rightarrow P^{*} \stackrel{\iota^{*}}{\rightarrow} Q^{*}$.

If $Q^{\prime}$ is a torus, by [4, Proposition 1.3] there exists a flasque resolution of $Q^{\prime}$

$$
\kappa: \quad 1 \longrightarrow S^{\prime} \longrightarrow P^{\prime} \stackrel{\rho}{\longrightarrow} Q^{\prime} \longrightarrow 1
$$

i.e. a short exact sequence where $P^{\prime}$ is a quasi-split torus and $S^{\prime}$ is a flasque torus. Using the same argument above we can show that the conditions in Theorem 4.1 are also equivalent to

(d) $f$ factors through $\rho: P^{\prime} \rightarrow Q^{\prime}$.

The following corollary shows that $e(Q)$ can be computed in many different ways.

Corollary 4.3. The following numbers are the same:

(i) $e(Q)$, the period of a generic $Q$-torsor.

(ii) $n_{1}:=$ the smallest positive integer $n$ such that the morphism $Q \stackrel{n}{\longrightarrow} Q$ factors through $\iota: Q \rightarrow P$.

(iii) The order of the class of $\chi$ in $\operatorname{Ext}_{G}^{1}\left(Q^{*}, S^{*}\right)$.

(iv) $n_{2}:=$ the smallest positive integer $n$ such that the morphism $Q \stackrel{n}{\longrightarrow} Q$ factors through some quasi-split torus.

If $Q$ is a torus, then $Q$ has a flasque resolution $\kappa$, and the above numbers are also equal to

(v) $n_{3}:=$ the smallest positive integer $n$ such that the morphism $Q \stackrel{n}{\longrightarrow} Q$ factors through $\rho: P^{\prime} \rightarrow Q$.

(vi) The order of the class of $\kappa$ in $\operatorname{Ext}_{G}^{1}\left(S^{* *}, Q^{*}\right)$.

Proof. First, it is clear that $n_{1}$ is equal to the order of the class of $\chi$. Then by the equivalence of (a) and (b) in Theorem 4.1 we have $n_{1}=n_{2}$. Finally, recall that $e(Q)$ is equal to the smallest integer $n$ such that $H^{1}(K, Q) \stackrel{n}{\longrightarrow} H^{1}(K, Q)$ is the zero homomorphism for every field extension $K / F$, therefore by Theorem 4.1 again we get $e(Q)=n_{1}=n_{2}$.

If $Q$ is a torus, by Remark 4.2 we see that $n_{2}=n_{3}$, which is also equal to the order of class of $\kappa$.

Example 4.4. Let $Q$ be a group of multiplicative type. Then $e(Q)=1$ if and only if $Q$ is a direct summand of a quasi-split torus, i.e. if and only if $Q$ is an invertible torus.

Example 4.5. Let $L / F$ be a finite Galois extension with Galois group $\operatorname{Gal}(L / F)=$ $G$. Consider the norm 1 torus $Q=R_{L / F}^{(1)}\left(\mathbb{G}_{m, L}\right)$, i.e. the torus defined by the short exact sequence

$$
\chi: \quad 1 \longrightarrow Q \longrightarrow R_{L / F}\left(\mathbb{G}_{m, L}\right) \stackrel{N}{\longrightarrow} \mathbb{G}_{m} \longrightarrow 1
$$

where $N$ is the norm map. Notice that the above exact sequence is a coflasque resolution of $Q$, hence $e(Q)$ is equal to the order of the class of the following exact 
sequence of character lattices (also denoted by $\chi$ )

$$
\chi: \quad 0 \longrightarrow \mathbb{Z} \stackrel{N}{\longrightarrow} \mathbb{Z}[G] \longrightarrow 0
$$

in $\operatorname{Ext}_{G}^{1}\left(Q^{*}, \mathbb{Z}\right)$. The norm map of character lattices $N: \mathbb{Z} \rightarrow \mathbb{Z}[G]$ is given by $N(1)=\sum_{g \in G} g$. It is then easy to verify that $e(Q)=$ order $([\chi])=|G|=[L: F]$.

\section{Relationships AMONG $e(W), e(T)$ And $e(Q)$}

For any group of multiplicative type $Q$, we have an exact sequence

$$
\zeta: \quad 1 \longrightarrow T \longrightarrow Q \longrightarrow W \longrightarrow 1
$$

where $T$ is a torus and $W$ is finite. The existence of the exact sequence $\zeta$ comes from the fact that the $\mathbb{Z}$-torsion elements of $Q^{*}$ form a $G$-submodule $\left(W^{*}\right)$ of $Q^{*}$.

Lemma 5.1. $e(W)=$ exponent of $W$.

Proof. Denote the exponent of $W$ by $n$. Since $W^{n}=1$, the homomorphism $H^{1}(K, W) \stackrel{n}{\longrightarrow} H^{1}(K, W)$ factors through $H^{1}(K, 1)=0$ for every field extension $K / F$. Hence $e(W) \mid n$.

Recall that $L$ is a splitting field of $W$, so $W_{L}=\mu_{n_{1}} \times \cdots \times \mu_{n_{r}}$ for some positive integers $n_{1}, \ldots, n_{r}$, where the least common multiple $\operatorname{lcm}\left\{n_{1}, \ldots, n_{r}\right\}$ of $n_{1}, \ldots, n_{r}$ is equal to $n$. Then for every field extension $K / L$,

$$
\begin{aligned}
H^{1}\left(K, W_{L}\right) & =H^{1}\left(K, \mu_{n_{1}}\right) \times \cdots \times H^{1}\left(K, \mu_{n_{r}}\right) \\
& =K^{\times} /\left(K^{\times}\right)^{n_{1}} \times \cdots \times K^{\times} /\left(K^{\times}\right)^{n_{r}} .
\end{aligned}
$$

Therefore $n=\operatorname{lcm}\left\{n_{1}, \ldots, n_{r}\right\}=e\left(W_{L}\right) \mid e(W)$ by Proposition 2.1.

Remark 5.2. Lemma 5.1 can also proved by using Corollary 4.3. First, $e(W)$ is the smallest positive integer $n$ such that the morphism $W^{*} \stackrel{n}{\longrightarrow} W^{*}$ factors through a permutation module. Since $W^{*}$ is finite and any permutation module is $\mathbb{Z}$-torsion free, $e(W)$ equals the smallest integer $n$ such that $n w=0$ for every $w \in W^{*}$, which is just the exponent of $W$.

Example 5.3. Let $Q$ be the group scheme of $n$-th roots of unity $\mu_{n}$ over $F$, where $\operatorname{char}(F)$ does not divide $n$. The exact sequence

$$
\chi: \quad 1 \longrightarrow \mu_{n} \longrightarrow \mathbb{G}_{m} \stackrel{n}{\longrightarrow} \mathbb{G}_{m} \longrightarrow 1
$$

is a coflasque resolution of $Q$. It is known that $\operatorname{Ext}_{\mathbb{Z}}^{1}(\mathbb{Z} / n \mathbb{Z}, \mathbb{Z}) \cong \mathbb{Z} / n \mathbb{Z}$, and $[\chi]$ is the canonical generator of $\operatorname{Ext}_{\mathbb{Z}}^{1}(\mathbb{Z} / n \mathbb{Z}, \mathbb{Z})$. Hence we recover the result $e\left(\mu_{n}\right)=n$.

Next we are going to find a lower bound for $e(Q)$ in terms of $e(T)$ and $e(W)$.

Lemma 5.4. $e(W) \mid e(Q)$.

Proof. Since $L$ is a splitting field of $Q$, we have $Q_{L}=T_{L} \times W_{L}$ and $T_{L}$ is a split torus. Therefore $e\left(W_{L}\right)=e\left(Q_{L}\right)$. It remains to observe that $e(W)=e\left(W_{L}\right)$ by Lemma 5.1, and $e\left(Q_{L}\right) \mid e(Q)$ by Proposition 2.1.

Lemma 5.5. $e(T) \mid e(Q)$. 
Proof. By Theorem $4.1 Q^{*} \stackrel{e(Q)}{\longrightarrow} Q^{*}$ factors through $\iota^{*}: P^{*} \rightarrow Q^{*}$. Since $P^{*}$ is a permutation module, it is $\mathbb{Z}$-torsion free and the homomorphism $Q^{*} \rightarrow P^{*}$ factors through $T^{*}$. This is illustrated in the following commutative diagram

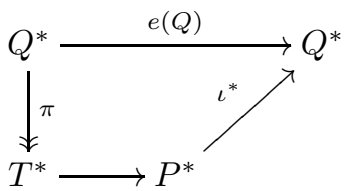

Then the composition $T^{*} \longrightarrow P^{*} \stackrel{\iota^{*}}{\longrightarrow} Q^{*} \stackrel{\pi}{\longrightarrow} T^{*}$ is just the homomorphism of multiplication by $e(Q)$. Therefore $e(T) \mid e(Q)$.

Corollary 5.6. $\operatorname{lcm}\{e(T), e(W)\} \mid e(Q)$.

Let $\lambda$ be the order of $[\zeta]$ in $\operatorname{Ext}_{G}^{1}\left(T^{*}, W^{*}\right)$. Then $\lambda$ is the smallest positive integer such that $T^{*} \stackrel{\lambda}{\longrightarrow} T^{*}$ factors through $Q^{*}$,

$$
T^{*} \stackrel{\psi}{\longrightarrow} Q^{*} \longrightarrow T^{*}
$$

for some $\psi: T^{*} \rightarrow Q^{*}$. Since $Q^{*}=T^{*} \oplus W^{*}$ as an abelian group, we can write every element in $Q^{*}$ in the form $(x, y)$ where $x \in T^{*}, y \in W^{*}$.

Lemma 5.7. $\psi$ can be chosen so that $\psi(x)=(\lambda x, 0)$ for every $x \in T^{*}$.

Proof. First note that $T^{*}$ is isomorphic to $\lambda T^{*}$ as a $G$-module, because $T^{*}$ is $\mathbb{Z}$-free. Consider the following exact sequence of $G$-modules

$$
0 \longrightarrow W^{*} \longrightarrow \lambda Q^{*}+W^{*} \longrightarrow \lambda T^{*} \longrightarrow 0
$$

where $\lambda Q^{*}+W^{*}$ is a submodule of $Q^{*}$, and the homomorphisms are the canonical ones. The composition

$$
\lambda T^{*} \stackrel{\sim}{\longrightarrow} T^{*} \stackrel{\psi}{\longrightarrow} \lambda Q^{*}+W^{*}
$$

is a splitting homomorphism for (3). Therefore $\lambda Q^{*}+W^{*}$ is isomorphism to $\lambda T^{*} \oplus$ $W^{*}$ as $G$-modules.

Proposition 5.8. $\operatorname{lcm}\{e(T), e(W)\}|e(Q)| \operatorname{lcm}\{\lambda e(T), e(W)\}$.

Proof. By Corollary 5.6 it suffices to prove the second divisibility. Choose $\psi$ such that $\psi(x)=(\lambda x, 0)$ for every $x \in T^{*}$. Let $n=\operatorname{lcm}\left\{e(T), \frac{e(W)}{\lambda}\right\}$, where $\lambda$ divides $e(W)$ because $\lambda$ is also the smallest positive integer such that $W^{*} \stackrel{\lambda}{\longrightarrow} W^{*}$ factors through $Q^{*}$. Then we consider the composition

$$
\begin{aligned}
& \beta: \quad Q^{*} \longrightarrow T^{*} \stackrel{n}{\longrightarrow} T^{*} \stackrel{\psi}{\longrightarrow} Q^{*} \\
& (x, y) \longmapsto x \longmapsto n x \longmapsto(\lambda n x, 0) .
\end{aligned}
$$

Since $e(W)$ is equal to the exponent of $W^{*}, \beta$ is just the endomorphism of $Q^{*}$ given by multiplication by $\lambda n$. By Theorem 4.1, $T^{*} \stackrel{n}{\longrightarrow} T^{*}$ factors through a permutation module. Therefore $\beta$ also factors through a permutation module, and it implies that

$$
e(Q) \mid \lambda n=\operatorname{lcm}\{\lambda e(T), e(W)\} .
$$

The following examples show that the inequalities in Proposition 5.8 are sharp. 
Example 5.9. Let $L / F$ be a quadratic field extension and $\operatorname{char}(F) \neq 2$, and let $\sigma$ be the generator of $G=\operatorname{Gal}(L / F)$. Consider the group of multiplicative type $Q$ given by $Q^{*}=\mathbb{Z} \oplus \mathbb{Z} / n \mathbb{Z}$ (as abelian group) with $G$-action $\sigma(a, b)=(-a, a+b)$, where $\operatorname{char}(F)$ does not divide $n$. By [7, Example 4.1] $e(T)=[L: F]=2$, and by Lemma $5.1 e(W)=n$.

By using [4, Lemma 0.6] we can construct a coflasque resolution of $Q^{*}$ explicitly and show that $e(Q)=2 n$. In particular, if $n=2$, then $\lambda=2$ also and we have $\operatorname{lcm}\{\lambda e(T), e(W)\}=4=e(Q)>\operatorname{lcm}\{e(T), e(W)\}$.

Example 5.10. Let $L / F$ be a field extension such that $G=\operatorname{Gal}(L / F)$ is a cyclic group of order 4 , for example $F=\mathbb{Q}(i)$ and $L=\mathbb{Q}\left(\xi_{16}\right)$, and let $\sigma$ be a generator of $G$. Let $T$ be the torus given by

$$
1 \longrightarrow \mathbb{G}_{m} \longrightarrow R_{L / F}\left(\mathbb{G}_{m}\right) \longrightarrow T \longrightarrow 1 \text {. }
$$

Then $T^{*}$ is (isomorphic to) the kernel of the augmentation map $\mathbb{Z}[G] \rightarrow \mathbb{Z}$. As an abelian group $T^{*}$ is generated by $a:=1-\sigma, b:=1-\sigma^{2}, c:=1-\sigma^{3}$.

Consider $Q^{*}=T^{*} \oplus \mathbb{Z} / 2 \mathbb{Z} \oplus \mathbb{Z} / 2 \mathbb{Z}$ (as abelian group) with $G$-action

$$
\sigma(x a+y b+z c, \bar{u}, \bar{v})=((-x-y-z) a+x b+y c, \bar{x}+\bar{y}+\bar{v}, \bar{u})
$$

for every $x, y, z \in \mathbb{Z}$ and for every $\bar{u}, \bar{v} \in \mathbb{Z} / 2 \mathbb{Z}$. By direction verification

$$
0 \longrightarrow \mathbb{Z} / 2 \mathbb{Z} \oplus \mathbb{Z} / 2 \mathbb{Z} \longrightarrow Q^{*} \longrightarrow T^{*} \longrightarrow 0
$$

does not split, hence $\lambda=2$. By $[7$, Example 4.1] $e(T)=[L: F]=4$. We claim that $e(Q)=4$ also by noting that $Q^{*} \stackrel{4}{\longrightarrow} Q^{*}$ factors as

$$
Q^{*} \longrightarrow T^{*} \stackrel{i}{\longrightarrow} \mathbb{Z}[G] \stackrel{\phi}{\longrightarrow} Q^{*}
$$

where $i$ is the inclusion, and $\phi$ is given by $\phi(1)=(a+b+c, 0,0)$. Hence $\operatorname{lcm}\{e(T), e(W)\}=$ $4=e(Q)<\operatorname{lcm}\{\lambda e(T), e(W)\}$.

\section{Application}

In the proof of Lemma 5.5 we have shown that $Q^{*} \stackrel{e(Q)}{\longrightarrow} Q^{*}$ factors as

$$
Q^{*} \stackrel{\pi}{\longrightarrow} T^{*} \longrightarrow P^{*} \longrightarrow Q^{*}
$$

where $P^{*}$ is a permutation module. If we write $Q^{*}=T^{*} \oplus W^{*}$ (as an abelian group), the composition homomorphism $T^{*} \rightarrow Q^{*}$ above is injective as it is given by $x \mapsto(e(Q) \cdot x, 0)$. Let $Z$ be the group of multiplicative type defined by $Z^{*}=$ $Q^{*} / \operatorname{im}\left(T^{*}\right)$, and we have an exact sequence

$$
1 \longrightarrow Z \longrightarrow Q \longrightarrow T \longrightarrow 1 \text {. }
$$

Passing to cohomology we obtain

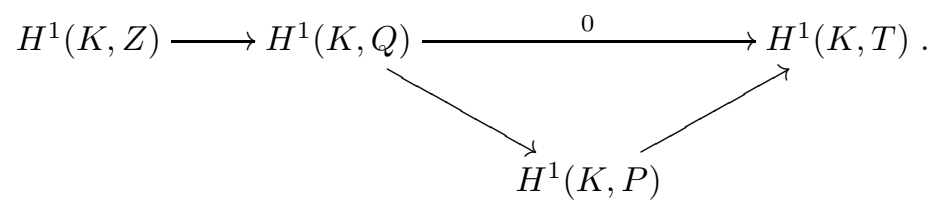

Therefore $Z$ is a finite subgroup of $Q$, with exponent $e(Q)$, such that the natural map $H^{1}(K, Z) \rightarrow H^{1}(K, Q)$ is surjective for every field extension $K / F$. 
The following proposition shows that the exponent of any finite subgroup of $Q$ with such property must be at least $e(Q)$.

Proposition 6.1. Let $Q$ be a group of multiplicative type, and $Z$ be any finite subgroup of $Q$ such that $H^{1}(K, Z) \rightarrow H^{1}(K, Q)$ is surjective for every field extension $K / F$. Then $e(Q)$ divides the exponent of $Z$.

Proof. First we recall that by Lemma 5.1 the exponent of the finite group $Z$ is equal to $e(Z)$. For every field extension $K / F, e(Z) \cdot H^{1}(K, Z)=0$. By assumption $H^{1}(K, Z) \rightarrow H^{1}(K, Q)$ is surjective, hence $e(Z) \cdot H^{1}(K, Q)=0$ also. It follows that $e(Q)$ divides $e(Z)$.

\section{ACKNOWLEDGEMENTS}

The author would like to thank his adviser Alexander Merkurjev for his help.

\section{REFERENCES}

[1] G. Berhuy and G. Favi, Essential dimension: a functorial point of view (after A. Merkurjev), Documenta Math. 8 (2003), 279-330.

[2] A. Borel, Linear Algebraic Groups, Graduate Texts in Mathematics 126, 2nd Enlarged Edition, Springer-Verlag, New York, 1991.

[3] J.-L. Colliot-Thélène and J.-J. Sansuc, La R-équivalence sur les tores, Ann. Sci. Éc. Norm. Sup. (4) 10 no. 2 (1977), 175-229.

[4] J.-L. Colliot-Thélène and J.-J. Sansuc, Principal Homogeneous Spaces under Flasque Tori: Applications, Journal of Algebra 106 (1987), 148-225.

[5] S. Garibaldi, A. Merkurjev, and J.-P. Serre, Cohomological Invariants in Galois Cohomology, University Lecture Series 28, American Mathematical Society, Providence, RI, 2003.

[6] P. Gille, Torseurs sur la droite affine et R-équivalence, Orsay (1994), Author's website.

[7] A. S. Merkurjev, Periods of principal homogeneous spaces of algebraic tori, Vestnik St. Petersburg University, Mathematics 43 no. 1 (2010), 39-43.

Department of Mathematics, University of California, Los Angeles, CA 90095-1555, USA

E-mail address: wanshunwong@gmail.com 